\title{
Índice general de los números 61 a 70 .
}

\section{(General Index of Issues 61-70)}

Achoy Solano, Daniela. «La implementación del aprendizaje de la gramática por descubrimiento guiado», Letras 68(2020) 143-173. Aguilar-Amat, Anna. «Compensación humana de déficits de la traducción automática», Letras 70 (2021) 131-167.

Alfaro Vargas, Roy. «Soumission: una fenomenología de la academia», Letras 64 (2018) 131-147.

Altamirano Alvarado, Jorge. «Leyendas y cuentos para combinar cultura y competencia lingüística», Letras 68 (2020) 107-141. Álvarez Garay, Estella. «L'écriture créative comme moyen pour stimuler la production écrite en FLE», Letras 69 (2021) 103-122. Argüello Borbón, Sandra. «Recalcitrant Framing in Kate Chopin's "Her Letters"», Letras 65 (2019) 93-106.

Baltodano Román, Gabriel. «Candice Lin: paisaje, territorio y fronteras», Letras 61 (2017) 69-90.

Baltodano Román, Gabriel. «La modernización de la risa en Costa Rica: Jiménez y Marín Cañas», Letras 68 (2020) 63-83.

Baltodano Román, Gabriel. «Norma política y enfermedad en Patria o muerte, de Alberto Barrera Tyszka», Letras 65 (2019) 39-59. Baltodano Román, Gabriel. «Subjetividad y subordinación en La hora de la estrella, de Clarice Lispector», Letras 66 (2019) 47-73.

Barquero, Luis. «Costa Rican Students' Mindsets Toward Studying English», Letras 65 (2019) 129-62.

Barquero Matamoros, Daniela. «Polifonía de lo inhumano: Delirio, de Laura Restrepo», Letras 69 (2021) 32-59.

1 Se clasifica según el apellido de todos los autores. Cuando hay entradas con dos autores, ambos aparecen según sendos apellidos. 
Barth Arroyo, Jorge Pablo. «Échelles bipolaires et représentations culturelles des élèves», Letras 63 (2018) 131-155.

Beltrán Almería, Luis. «Humorismo, la cultura de la risa», Letras 68 (2020) 15-36.

Beltrán Almería, Luis. «La novela, género literario», Letras 66 (2019) $13-45$.

Bolaños Villalobos, Isabel Cristina. «Proceso de declive del buglere», Letras 67 (2020) 91-111.

Bonilla Corrales, Karen. «El pensamiento centroamericano del siglo XIX: política y educación», Letras 62 (2017) 47-72.

Bonilla Navarro, José Francisco. «La utopía conceptual feminista y el discurso literario: dos novelas de Roxana Pinto», Letras 63 (2018) 59-78.

Borda, José Joaquín. «Provincialismos de Costa Rica», Letras 67 (2020) 205-209.

Burgos Pérez, Fernando. «El devenir inhumano en El desván, De Ramón H. Jurado», Letras 64 (2018) 27-49.

Camacho Arias, Ariadne. «Erotismo indio en "Las mareas vuelven de noche", de Yolanda Oreamuno», Letras 64 (2018) 73-85.

Camacho Guzmán, Gustavo. «El pensamiento centroamericano del siglo XIX: política y educación», Letras 62 (2017) 47-72.

Camacho Guzmán, Gustavo. «El teatro centroamericano del ocaso colonial. Joaquín de Oreamuno y Víctor de la Guardia», Letras 65 (2019) 13-37.

Camacho Guzmán, Gustavo. «La novela centroamericana sobre el migrante: Las murallas y Al otro lado del San Juan», Letras 68 (2020) 85-104.

Camacho Guzmán, Gustavo. «La nueva novela histórica y la construcción de la feminidad en una novela de Gioconda Belli», Letras 63 (2018) 79-91.

Camacho Guzmán, Gustavo. «La representación de la cultura centroamericana en el relato de viajes de Wilhem Marr», Letras 70 (2021) $15-41$. 
Camacho Guzmán, Gustavo. «Referencialidad e ironía en El crimen de Alberto Lobo, de Gonzalo Chacón Trejos», Letras 61 (2017) 39-67.

Castro-García, Damaris. «Teaching Methodology and Receptive Vocabulary Measures», Letras 66 (2019) 119-143.

Chaves Fernández, Lindsay. «Efectos colaterales de los exámenes orales por tribunal», Letras 68 (2020) 199-238.

Chaves Fernández; Lindsay. «Traducción al inglés de "Abbott y Costello", de Sergio Ramírez: una perspectiva terminológica», Letras 70 (2021) 169-198.

Chaves Carballo, Olga. «Oferta educativa para un bachillerato en mandarín», Letras 63 (2018) 157-182.

Chaves Jiménez, Óscar. «Students’ Insights on E-Portfolios in English for Specific Purposes», Letras 66 (2019) 145-63.

Cordero Badilla, Damaris. «Errores léxicos en la producción escrita de estudiantes de ILE», Letras 68 (2020) 175-198.

Cordero Cordero, José Enrique. «Mundos antagónicos en "Bananos y hombres", de Carmen Lyra», Letras 69 (2021) 61-79.

Costa Morales, Karina «Des gallicismes dans l'espagnol costaricien», Letras 70 (2021) 79-104.

Fallas Escobar, Christian. «Reading As Onlookers or As Critical Participants?», Letras 64 (2018) 169-189.

Gapper, Sherry E. «Historia y proyecciones de una revista dedicada a la palabra», Letras 70 (2021) 201-224.

Giangiulio Lobo, Alejandra. «Evaluating Basic Grammar Projects, Using the SAMR Model», Letras 61 (2017) 123-151.

González Hernández, Carlos Andrés. «Representaciones de la obesidad en la cuentística de Franz Galich y de Carlos Velázquez», Letras 67 (2020) 39-58.

González Sánchez, Esteban. «Donde no estén ustedes. Prisma de la narrativa de Horacio Castellanos Moya», Letras 63 (2018)29-40. 
Guzmán Arce, Natin. «El aprendizaje móvil: enfoque integrador para aumentar la participación de los estudiantes», Letras 67 (2020) 145-207.

Gyulamiryan, Tatevik. «Sancho reacentuado y su teoría de la mente», Letras 69 (2021) 13-29.

Hernández Carmona, Luis Javier. «El ensayo lírico como experiencia estética-hermenéutica», Letras 61 (2017) 13-37.

Herrera Valenciano, Minor. «Represión, acción y persuasión en

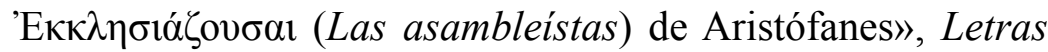
62 (2017) 93-115.

Herrera Valenciano, Minor. «Tradición clásica y utopía en La caída del águila, de Carlos Gagini», Letras 64 (2018) 51-72.

Jiménez Murillo, Juan Carlos. «Dualité ou triplication du protagoniste dans le conte "Histoire d'un mort racontée par lui-même", d'A. Dumas?», Letras 70 (2021) 43-75.

Jiménez Murillo, Juan Carlos. «Identifier, déchiffrer et interpréter le code énigmatique dans l'œuvre de Jean Ray: vers une lecture policière du conte «Le Gardien du Cimetière»»", Letras 62 (2017) 117-149.

Jiménez Murillo, Juan Carlos. «Le jeu conflictuel du dédoublement dans "J'ai tué Alfred Heavenrock" de Jean Ray: un paradoxe identitaire entre le héros et son Doppelgänger», Letras 65 (2019) 61-92.

Lara Jiménez, Rocío. «Evaluating Basic Grammar Projects, Using the SAMR Model», Letras 61 (2017) 123-151.

Leis, Adrián. «Costa Rican Students' Mindsets Toward Studying English», Letras 65 (2019) 129-62.

López Estrada, Patricia. «Students' Insights on E-Portfolios in English for Specific Purposes», Letras 66 (2019) 145-63.

Marín Esquivel, Rebeca. «Acercamiento a la morfología verbal del español guaymí costarricense», Letras 69 (2021) 83-100.

Marín Esquivel, Rebeca. «Indicios del desplazamiento del guaymí en historias sociolingüísticas», Letras 66 (2019) 165-191. 
Martínez Arbelaiz, Asunción. «La narrativa reflexiva como forma de evaluación programática», Letras 61 (2017) 93-121.

Méndez, María Oliva. «Nación y utopía en Cuadros de costumbres guatemaltecas, de José Milla», Letras 62 (2017) 13-30.

Molina Morales, Guillermo. «Risa y poesía. Cinco momentos centroamericanos», Letras 68 (2020) 37-61.

Monge, Carlos Francisco. «En el territorio de la incertidumbre: entrevista, poemas y comentarios de autolectura», Letras 69 (2021) 141-173.

Montenegro Bonilla, Joe. «The American Past and Present: A New Historicist Approach to The Adventures of Tom Sawyer», Letras 64 (2018) 109-112.

Núñez, Vianey Martín. «Errores léxicos en la producción escrita de estudiantes de ILE», Letras 68 (2020) 175-198.

Núñez Alvarado, Viviana. «Las partículas subjetivas del español centroamericano: indagaciones preliminares», Letras 64 (2018) 151-165.

Olivares Garita, Cinthya. «Leyendas y cuentos para combinar cultura y competencia lingüística», Letras 68 (2020) 107-141.

Ovares, Flora. «La sirvienta y el luchador. Una interpretación», Letras 63 (2018) 15-27.

Paniagua, Carlos. «Del acontecimiento teatral al papel: Rabinal Achì», Letras 64 (2018) 87-107.

Porras Núñez, Mariela. «Reading As Onlookers or As Critical Participants?», Letras 64 (2018) 169-189.

Ramírez Villalobos, Gretel. «La modernización de la risa en Costa Rica: Jiménez y Marín Cañas», Letras 68 (2020) 63-83.

Ramírez Villalobos, Gretel. «Norma política y enfermedad en Patria o muerte, de Alberto Barrera Tyszka», Letras 65 (2019) 39-59. Ramírez Villalobos, Gretel. «Subjetividad y subordinación en La hora de la estrella, de Clarice Lispector», Letras 66 (2019) 47-73.

Rivera Cerdas, Gineth Tatiana. «Mundos antagónicos en "Bananos y hombres", de Carmen Lyra», Letras 69 (2021) 61-79. 
Robles Murillo, Keylor. «La mujer modélica en Movimiento perpetuo, de Augusto Monterroso», Letras 67 (2020) 15-38.

Rojas G., Margarita. «La sirvienta y el luchador. Una interpretación», Letras 63 (2018) 15-27.

Rojas Ugalde, Ana. «Enhancing Language Learning and Acquisition by Implementing Extensive Reading», Letras 69 (2021) 123-137.

Sánchez Murillo, Julio. «Des gallicismes dans l'espagnol costaricien», Letras 70 (2021) 79-104.

Sánchez Murillo, Julio. «La enseñanza de lenguas extranjeras en universidades públicas de América», Letras 63 (2018) 95-130. Sánchez Santiago, Tomás. «En el territorio de la incertidumbre: entrevista, poemas y comentarios de autolectura», Letras 69 (2021) 141-173.

Schaumloeffel, Marco A. «Papiamento y la conexión brasileña establecida mediante los judíos sefardíes», Letras 67 (2020) 75-89.

Sevilla Morales, Henry. «Current Complexities of English Teaching in Costa Rica», Letras 61 (2017) 153-178.

Sevilla Morales, Henry. «Efectos colaterales de los exámenes orales por tribunal», Letras 68 (2020) 199-238.

Sevilla Morales, Henry. «Lo bueno, lo malo y lo impensable de la autonomía en el aprendizaje de ILE», Letras 67 (2020) 115-144. Sevilla Morales, Henry. «Traducción al inglés de "Abbott y Costello", de Sergio Ramírez: una perspectiva terminológica», Letras 70 (2021) 169-198.

Shiau-Bo, Liang. «Función de los diarios en Elzorro de arribay elzorro de abajo, de José María Arguedas», Letras 66 (2019) 91-115.

Solano Moraga, Sigrid. «Erotismo indio en "Las mareas vuelven de noche", de Yolanda Oreamuno», Letras 64 (2018) 73-85.

Solano Moraga, Sigrid. «La enajenación en El circo del deseo, de Cirus Piedra», Letras 66 (2019) 75-89.

Sun, Junchi. «Personalidad dual y esquizofrenia en personajes de literatura centroamericana contemporánea», Letras 67 (2020) 59-71. 
Torres-Hostench, Olga. «Compensación humana de déficits de la traducción automática», Letras 70 (2021) 131-167.

Valerio Arce, José Pablo. «Polifonía de lo inhumano: Delirio, de Laura Restrepo», Letras 69 (2021) 32-59.

Valverde Jara, Xinia. «Las notas al pie en la traducción de Fifty Shades», Letras 61 (2017) 181-202.

Vargas Barquero, Vivian. «El aprendizaje móvil: enfoque integrador para aumentar la participación de los estudiantes», Letras 67 (2020) 145-207.

Vargas Barquero, Vivian. «Enhancing Language Learning andAcquisition by Implementing Extensive Reading», Letras 69 (2021) 123-137. Vargas Barquero, Vivian. «Oferta educativa para un bachillerato en mandarín», Letras 63 (2018) 157-182.

Vargas Gómez, Francisco Javier. «Hablo, luego existo», Letras 67 (2020) 179-209.

Vargas Gómez, Francisco Javier. «Historia y proyecciones de una revista dedicada a la palabra», Letras 70 (2021) 201-224.

Vargas Murillo, María Gabriela. «Échelles bipolaires et représentations culturelles des élèves», Letras 63 (2018) 131-155.

Vargas Vargas, José Ángel. «La fugitiva. Libertad personal, exilio e indiferencia», Letras 62 (2017) 73-89.

Vargas Vargas, José Ángel. «Migración y deconstrucción del imaginario costarricense en "Abbott y Costello", de Sergio Ramírez», Letras 64 (2018) 13-26.

Vega Chavarría, Melany. «Fonética y fonología de las consonantes oclusivas del guaymí hablado en Costa Rica», Letras 70 (2021) 105-128.

Villafuerte Guzmán, Félix Javier. «El cristiano errante, de Antonio de Irisarri, y la literatura satírica en Centroamérica», Letras 62 (2017) 31-46.

Villalobos Varela, Breda. «Un mortífero viaje en un cuento de Horacio Castellanos Moya», Letras 63 (2018) 41-55.

Yang, Ming. «Introducción de verbos inacusativos en la clase de español como lengua extranjera», Letras 65 (2019) 109-128. 
